\title{
Effect of texture on the magnetostriction of grain-aligned composite Terfenol-D
}

\author{
Oh Yeoul Kwon, Hee Yeoun Kim, and Soon Hyung Hong a) \\ Department of Materials Science and Engineering, Korea Advanced Institute of Science and Technology, \\ 373-1 Guseong-Dong, Yuseong-Gu, Daejeon 305-701, Korea \\ De Rac Son \\ Department of Physics, Hannam University, 133 Ojung-Dong, Daeduk-Gu, Daejeon 306-791, Korea
}

(Received 23 February 2006; accepted 18 October 2006; published online 20 December 2006)

\begin{abstract}
Grain-aligned composite Terfenol-D was fabricated via unidirectional solidification, followed by the infiltration of epoxy. The fraction of the $R \mathrm{Fe}_{2}$ phase in composite Terfenol-D depends on the iron/rare earth $(\mathrm{RE}=\mathrm{Tb}, \mathrm{Dy})$ ratio of the as-grown Terfenol-D and the direction of the $R \mathrm{Fe}_{2}$ phase in the as-grown Terfenol-D changes from $\langle 110\rangle$ to $\langle 111\rangle$ with an increasing iron/rare earth ratio. For a series of grain-aligned composite Terfenol-D with different $R \mathrm{Fe}_{2}$ fractions, the magnetostriction was found to increase from 813 to $1013 \mathrm{ppm}$ with increases in the $R \mathrm{Fe}_{2}$ fraction. A model for the magnetostriction of grain-aligned composite Terfenol-D was developed based on the modified uniform strain condition. The modified uniform strain model was then used to predict the magnetostriction of grain-aligned composite Terfenol-D from the fraction of the $R \mathrm{Fe}_{2}$ phase, the elastic modulus for each crystal direction of the $R \mathrm{Fe}_{2}$ phase obtained in a texture analysis, and the saturation magnetostriction value of each preferred direction. The predicted magnetostriction of grain-aligned composite Terfenol-D, which considers the elastic modulus with respect to the crystal direction of the magnetostrictive phase, is in agreement with the experimental results. In conclusion, in grain-aligned composite Terfenol-D fabricated using the crystal growth technique the preferred direction should be considered for predicting the magnetostriction of grain-aligned composite Terfenol-D. (C) 2006 American Institute of Physics. [DOI: 10.1063/1.2402347]
\end{abstract}

\section{INTRODUCTION}

Magnetostrictive composites have interesting potential applications, such as high-frequency transducers or sensors, because they can reduce the eddy current loss and improve the fracture toughness compared to monolithic Terfenol-D. In general, magnetostrictive [1-3] composites can be fabricated by integrating magnetostrictive powders embedded in a nonmetallic matrix, such as epoxy or metallic matrix (Al, $\mathrm{Cu}$, or $\mathrm{Fe}$ ); on applying a magnetic field along the longitudinal direction of the composite, this results in an insulating layer between the particles. ${ }^{1,2}$ Although these [1-3] composites are based on magnetically chained particles producing a strain, they cannot avoid the demagnetizing effect and have much lower saturation magnetostriction than monolithic magnetostrictive materials. ${ }^{3-5}$

Previously, we reported the magnetostriction properties of the grain-aligned composite Terfenol-D, which consists of a unidirectionally solidified crystal instead of particles and is made using a composite technique. ${ }^{6,7}$ As the grain-aligned composite Terfenol-D consists of the preform, which maintains the bulk texture, and epoxy as an insulating layer, it is difficult to predict the magnetostriction of an epoxy-filled composite using a model based on magnetostrictive particles.

Herbst et $a l^{8}{ }^{8}$ reported the effective magnetostriction of

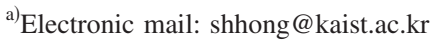

hot-pressed $\mathrm{SmFe}_{2} / \mathrm{Al}(\mathrm{Fe})$ composites with $\mathrm{SmFe}_{2}$ volume fractions of $f=0.2-0.8$ obtained from powdering annealed, single-phase ingots. They assumed that an elastically isotropic magnetostrictive sphere has a radius $R_{1}$ and saturation magnetostriction $\lambda_{s}$ embedded in an elastically nonmagnetostrictive medium. Nan ${ }^{9}$ studied the effective magnetostriction of the isotropic composite constituting a magnetostrictive particle and a nonmagnetostrictive phase using a Green function method. Chen et al. ${ }^{10}$ investigated the effect of the volume fraction of various matrix materials (glass, glass $+\mathrm{Al}$, $\mathrm{Fe}, \mathrm{Cu}$, or $\mathrm{CeFe}_{2}$ ) on the magnetostriction of composites containing magnetostrictive particles. Guo et al. ${ }^{11}$ studied the magnetostriction of glass, epoxy/Terfenol-D composites for $V_{f}$ values of Terfenol-D particles in the range of 0.1-0.82 and suggested a model to describe the $V_{f}$ dependence of magnetostriction and the coupling coefficient of the composite.

Since the reported magnetostrictive material model for a composite is based on the magnetostrictive particles, it is not necessary to consider the crystal orientation of the magnetostrictive phase. Therefore, relatively little research has examined the magnetostriction model of composite Terfenol-D, which maintains the texture of a unidirectionally solidified crystal. Here, we investigated the magnetostriction of grainaligned composite Terfenol-D and developed a model to describe both the texture fraction and elastic modulus change with texture depending on the magnetostriction of the magnetostrictive phase $\left(R \mathrm{Fe}_{2}\right)$. 


\section{EXPERIMENTAL PROCEDURES}

$\mathrm{Tb}_{0.33} \mathrm{Dy}_{0.67} \mathrm{Fe}_{\mathrm{y}}$ alloys with different chemical compositions $(y=1.10,1.36$, and 1.66) were prepared using arc melting under high-purity argon and were then cast into rods using a suction method after induction melting. These rods were then sealed into a quartz ampoule and grown to a length of $150 \mathrm{~mm}$ by zone melting at a growth rate of $70 \mu \mathrm{m} / \mathrm{s}$. For composite Terfenol-D, we first placed cylindrically machined samples (20 mm long and $6 \mathrm{~mm}$ in diameter) in a quartz ampoule containing quartz granules and sealed them at 360 Torr with purified argon to remove the eutectic phase from the as-grown Terfenol-D. On annealing at $1000{ }^{\circ} \mathrm{C}$, the eutectic phase, which has a low melting point $\left(\sim 890^{\circ} \mathrm{C}\right.$, although it differs slightly depending on the $\mathrm{Tb} / \mathrm{Dy}$ ratio), leaked from the sample because of the reaction between the quartz granules and the sample. Then, the porous preform Terfenol-D, which is composed of cellular-structured $R \mathrm{Fe}_{2}$ prepared by extracting the eutectic phase, was immersed in liquid epoxy at a temperature of $70{ }^{\circ} \mathrm{C}$, which has the lowest viscosity and pressurized with argon at 1200 Torr so that the porous preform with leaked channels was replaced with the epoxy. Finally, after curing the polymer infiltrated preform Terfenol-D, the grain-aligned composite Terfenol-D was finally fabricated using the unidirectional solidification technique.

The texture of the preform was measured by applying the Shultz reflection method using $\mathrm{Cu} K \alpha$ and an orientation distribution function (ODF) analysis was performed to determine the texture information for the magnetostrictive phase $\left(R \mathrm{Fe}_{2}\right)$ of the unidirectionally solidified crystal. Through ODF analysis, the volume fraction ${ }^{12}$ of each direction with orientation $g$ of the crystal axis with respect to a fixed sample coordinate system can be found from

$$
F(g)=(V / d V) / d g .
$$

Furthermore, the theoretical $E_{p}$ (Ref. 13) of preform Terfenol-D consisting of $R \mathrm{Fe}_{2}$ phase with a cubic structure is given by

$$
1 / E=S_{11}-2\left(S_{11}-S_{12}-1 / 2 S_{44}\right)\left(l_{1}^{2} l_{2}^{2}+l_{2}^{2} l_{3}^{2}+l_{3}^{2} l_{1}^{2}\right),
$$

where $s_{i j}$ and $l_{i}$ are the compliance and directional cosine between each direction and the crystal axis $(x, y, z)$, respectively. The fraction of $R \mathrm{Fe}_{2}$ phase in the composite was confirmed by measuring it with an image analyzer and determining the density. The magnetostrictive strain was measured along the longitudinal direction using a linear variable differential transform (LVDT) for each as-grown and grain-aligned composite Terfenol-D at room temperature. To measure the magnetostriction, a solenoid was used to provide a magnetic field of up to $80 \mathrm{kA} / \mathrm{m}$ and a compressive prestress ranging from 2 to $20 \mathrm{MPa}$ was applied along the rod axis of the sample. $^{14}$

\section{RESULTS AND DISCUSSION}

The magnetostriction model of grain-aligned composite Terfenol-D was based on the assumption of a uniform strain. Since in a preform of composite Terfenol-D prepared using unidirectional solidification the dendrite $R \mathrm{Fe}_{2}$ phase is ori- ented in the longitudinal direction and because Poisson's ratios ${ }^{15}$ of a preform Terfenol-D consisting of only the $R \mathrm{Fe}_{2}$ phase, and the epoxy in composite Terfenol-D are similar, it is reasonable to apply the uniform strain model. In the uniform strain model, the equilibrium of the forces in the longitudinal direction can be determined from

$$
\sigma_{c} A_{c}=\sigma_{R \mathrm{Fe}_{2}} A_{R \mathrm{Fe}_{2}}+\sigma_{e} A_{e},
$$

where $\sigma$ and $A$ are the stress and cross-sectional area, respectively. The subscripts $c, R \mathrm{Fe}_{2}$, and $e$ are the grain-aligned composite Terfenol-D, the magnetostrictive phase producing the magnetostriction, and epoxy with nonmagnetic properties, respectively. By inserting the strain of each component $\left(\varepsilon_{c}=\varepsilon_{R \mathrm{Fe}_{2}}=\varepsilon_{e}\right)$ in Eq. (3), we get

$$
E_{c}=E_{\mathrm{Re}_{2}} V_{R \mathrm{Fe}_{2}}+E_{e} V_{e},
$$

where $E_{c}, E_{R \mathrm{Fe}_{2}}$, and $E_{e}$ are the elastic modulus of composite Terfenol-D, the $R \mathrm{Fe}_{2}$ phase, and epoxy, respectively.

In this magnetostriction model, because the magnetostriction in the grain-aligned composite Terfenol-D depends only on the magnetostrictive phase of $R \mathrm{Fe}_{2}$, we assume that the overall stress of the composite Terfenol-D as it is magnetically saturated, is the product of the fraction of the $R \mathrm{Fe}_{2}$ phase showing magnetostriction and the stress exerted by the $R \mathrm{Fe}_{2}$ phase. Under this condition, we can obtain

$$
E_{c} \lambda_{c}=E_{R \mathrm{Fe}_{2}} V_{R \mathrm{Fe}_{2}} \lambda_{R \mathrm{Fe}_{2}}^{\mathrm{st}} .
$$

Since the grain-aligned composite Terfenol-D is based on preform Terfenol-D to maintain the texture of unidirectional solidification, Eq. (5) can be transformed into

$$
E_{c} \lambda_{c}=\sum_{i=0}^{n} E_{h_{i} k_{i} l_{i}}^{R \mathrm{Fe}_{2}} V_{h_{i} k_{i} l_{i}}^{R \mathrm{Fe}_{2}} \lambda_{h_{i} k_{i} l_{i}}^{\mathrm{st}},
$$

where $E_{h k l}$ and $V_{h k l}$ are the elastic modulus and volume fraction, respectively, for each direction of the preform Terfenol-D consisting of only the $R \mathrm{Fe}_{2}$ phase. Here, $\lambda_{h k l}$ is the saturation magnetostriction for each direction in the $R \mathrm{Fe}_{2}$ phase. When we substitute the expressions for the elastic modulus in Eq. (4), we obtain the magnetostriction limiting value for the composite Terfenol-D as follows:

$$
\lambda_{c}=\frac{\sum_{i=0}^{n} E_{h_{i} k_{i} l_{i}}^{R \mathrm{Fe}_{i}} V_{h_{i} k_{i} l_{i}}^{R \mathrm{Fe}_{i}} \sum_{h_{i} k_{i} l_{i}}^{\mathrm{st}} .}{E_{c}} .
$$

Since grains with different crystallographic orientations relative to the direction of measurement have different magnetostrictive properties, the various orientations in composite Terfenol-D consisting of a polycrystalline grown by zone melting should be considered to predict the effective magnetostriction of the grain-aligned composite Terfenol-D. Therefore, our magnetostriction model allows one to study the effect of the fraction of the magnetostrictive phase and the texture of the magnetostriction phase preserved during unidirectional solidification. The microstructure of grain-aligned composite Terfenol-D investigated in this model is given in Fig. 1, which shows that grain-aligned composite Terfenol-D is produced using the epoxy infiltration method. 


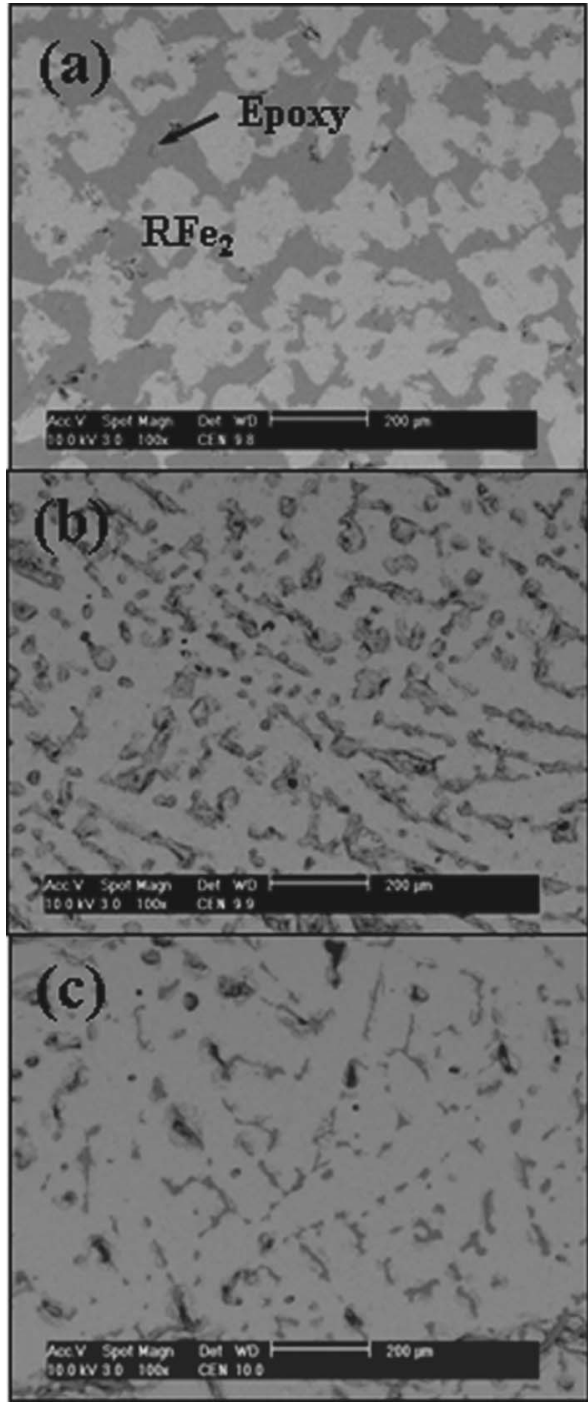

FIG. 1. Microstructures of grain-aligned composite Terfenol-D, following annealing and the infiltration of epoxy. Grain-aligned composite Terfenol-D with compositions of (a) $\mathrm{Tb}_{0.33} \mathrm{Dy}_{0.67} \mathrm{Fe}_{1.10}$, (b) $\mathrm{Tb}_{0.33} \mathrm{Dy}_{0.67} \mathrm{Fe}_{1.36}$, and (c) $\mathrm{Tb}_{0.33} \mathrm{Dy}_{0.67} \mathrm{Fe}_{1.66}$.

The normalized fraction for each preferred direction of the magnetostrictive $R \mathrm{Fe}_{2}$ phase in grain-aligned composite Terfenol-D, the orientation distribution function $f(g)$ and the calculated elastic modulus of grain-aligned composite Terfenol-D are shown in Table I. The ODF results in Table I are very useful because they provide quantitative information about the entire spread of orientations in the texture. Based on this ODF, the normalized volume of the orientation space can be calculated by dividing the orientation density of each preferred orientation by the total ODF value.

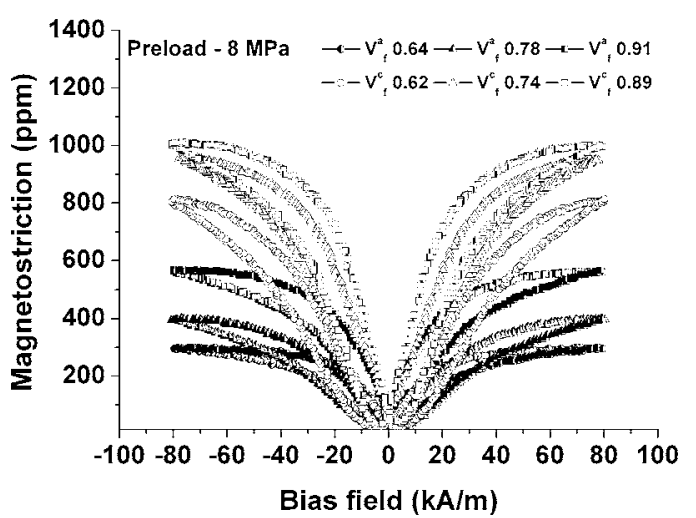

FIG. 2. Comparison of the magnetostriction of as-grown and grain-aligned composite Terfenol-D as a function of the bias field at $8 \mathrm{MPa}$. The superscripts $c$ and $a$ indicate composite and as-grown Terfenol-D, respectively.

In the elastic modulus calculation for grain-aligned composite Terfenol-D, the elastic modulus of the $R \mathrm{Fe}_{2}$ phase is calculated theoretically by summing the products of the calculated elastic modulus for each preferred direction and the normalized fraction of $R \mathrm{Fe}_{2}$ phase in each direction, while we use an elastic modulus value of $3.0 \mathrm{GPa}$ for the epoxy. The theoretical elastic modulus of the $R \mathrm{Fe}_{2}$ phase is calculated using the elastic stiffness constants of monolithic Terfenol-D. ${ }^{16}$ Moreover, when the $\mathrm{RE} / \mathrm{Fe}$ ratio increases from 1.10 to 1.66 , the preferred direction of the $R \mathrm{Fe}_{2}$ phase results in the transition from the $\langle 110\rangle$ to the $\langle 111\rangle$ direction.

The magnetostriction curves of the as-grown and grainaligned composite Terfenol-D are presented in Fig. 2. This shows that the maximum magnetostriction of composite Terfenol-D is proportional to the fraction of the $R \mathrm{Fe}_{2}$ phase. When we remove the eutectic phase from as-grown Terfenol-D and then substitute epoxy for the eutectic phase, the magnetostriction of the grain-aligned composite Terfenol-D improves significantly (it is about two times higher than the magnetostriction of as-grown Terfenol-D). The improvement in the magnetostriction in the composite is divided into two broad contributions: annealing and the removal of the eutectic phase with nonmagnetic properties. The effect of the former on the magnetostriction was reported by Verhoeven et al. ${ }^{17}$ and Mei et al. ${ }^{18}$ who found that the magnetostriction improved to about $20 \%$ compared to the as-grown Terfenol-D during heat treatment due to relief of the residual stress in the as-solidified materials. Since the effect of heat treatment on magnetostriction in Terfenol-D is not major, the greatest improvement in the magnetostriction of the grain-aligned composite Terfenol-D compared to as-

TABLE I. Magnetostrictive phase fraction and normalized $f(g)$ value for as-grown Terfenol-D and the predicted elastic modulus of grain-aligned composite Terfenol-D.

\begin{tabular}{|c|c|c|c|c|c|c|c|}
\hline \multirow[b]{2}{*}{ Composition } & \multicolumn{2}{|c|}{$\begin{array}{c}\text { Fraction of the } R \mathrm{Fe}_{2} \\
\text { phase }\end{array}$} & \multicolumn{4}{|c|}{$\begin{array}{c}\text { Normalized fraction of } R \mathrm{Fe}_{2} / f(\mathrm{~g}) \text { value for } \\
\text { each direction }\end{array}$} & \multirow{2}{*}{$\begin{array}{c}\text { Predicted elastic } \\
\text { modulus of } \\
\text { composite Terfenol-D } \\
(\mathrm{GPa})\end{array}$} \\
\hline & As grown & Composite & {$[110]$} & [113] & [112] & [111] & \\
\hline $\mathrm{Tb}_{0.33} \mathrm{Dy}_{0.67} \mathrm{Fe}_{1.10}$ & 0.64 & 0.62 & $0.52 / 5.6$ & $0.22 / 3.5$ & $0.26 / 3.5$ & $\cdots$ & 26 \\
\hline $\mathrm{Tb}_{0.33} \mathrm{Dy}_{0.67} \mathrm{Fe}_{1.36}$ & 0.78 & 0.74 & $0.45 / 6.3$ & $0.25 / 4.1$ & $0.30 / 4.0$ & $\cdots$ & 37 \\
\hline $\mathrm{Tb}_{0.33} \mathrm{Dy}_{0.67} \mathrm{Fe}_{1.66}$ & 0.91 & 0.89 & $0.56 / 14.8$ & $\cdots$ & $0.16 / 5.1$ & $0.28 / 7.8$ & 56 \\
\hline
\end{tabular}


TABLE II. The magnetostrictive properties of grain-aligned composite Terfenol-D. The maximum strain and $d_{33}$ were obtained from measurements at $80 \mathrm{kA} / \mathrm{m}$ under a preload of $8 \mathrm{MPa}$, and the coupling coefficient was determined by measuring the impedance (direct method) considering the mass distribution.

\begin{tabular}{|c|c|c|c|c|c|c|c|}
\hline \multirow[b]{3}{*}{ Composition } & \multicolumn{6}{|c|}{ Magnetostrictive properties $(80 \mathrm{kA} / \mathrm{m}, 8 \mathrm{MPa})$} & \multirow{3}{*}{$\begin{array}{c}\text { Predicted } \\
\text { magnetostriction } \\
\text { of composite } \\
\text { Terfenol-D } \\
(\mathrm{ppm})\end{array}$} \\
\hline & \multicolumn{2}{|c|}{ Max. strain (ppm) } & \multicolumn{2}{|c|}{$d_{33}(\mathrm{~nm} / \mathrm{A})$} & \multicolumn{2}{|c|}{$k_{33}^{\max }$} & \\
\hline & As grown & Composite & As grown & Composite & As grown & Composite & \\
\hline $\mathrm{Tb}_{0.33} \mathrm{Dy}_{0.67} \mathrm{Fe}_{1.10}$ & 299.8 & 813.3 & 6.6 & 15.3 & 0.22 & 0.34 & 839.8 \\
\hline $\mathrm{Tb}_{0.33} \mathrm{Dy}_{0.67} \mathrm{Fe}_{1.36}$ & 400.0 & 960.6 & 11.3 & 25.2 & 0.30 & 0.43 & 963.3 \\
\hline $\mathrm{Tb}_{0.33} \mathrm{Dy}_{0.67} \mathrm{Fe}_{1.66}$ & 560.6 & 1013 & 13.4 & 27.8 & 0.36 & 0.45 & 1045.4 \\
\hline
\end{tabular}

grown Terfenol-D at the same fraction of the $R \mathrm{Fe}_{2}$ phase is largely attributable to the absence of the eutectic phase, which inhibits the magnetization rotations.

The measured maximum magnetostriction of as-grown and grain-aligned composite Terfenol-D and the predicted magnetostriction for the composite are shown in Table II. For the predicted magnetostriction of grain-aligned composite Terfenol-D, we used the saturation magnetostrictive strains of each orientation studied by Teter et al. ${ }^{19}$ and Zhang et al. ${ }^{20}$

The magnetostriction model was developed as a function of the fraction of the magnetostrictive phase $\left(R \mathrm{Fe}_{2}\right)$ of grainaligned composite Terfenol-D; this model used the elastic modulus obtained in the texture analysis for the magnetostrictive phase and $3 \mathrm{GPa}$ for the epoxy. The results are compared to the experimental magnetostriction for the composite Terfenol-D in Fig. 3. In this figure, the open diamonds indicate the predicted magnetostriction based on the assumption of modified uniform strain. Furthermore, the dotted line shows the predictions for grain-aligned composite Terfenol-D under the uniform strain model, which does not consider the change in the elastic modulus with the change in the preferred direction of the crystal. Since several studies have reported that the preferred direction of monolithic Terfenol-D is $\langle 112\rangle,{ }^{18,19,21}$ in the uniform strain model, we assume that the crystal is completely oriented along the $\langle 112\rangle$

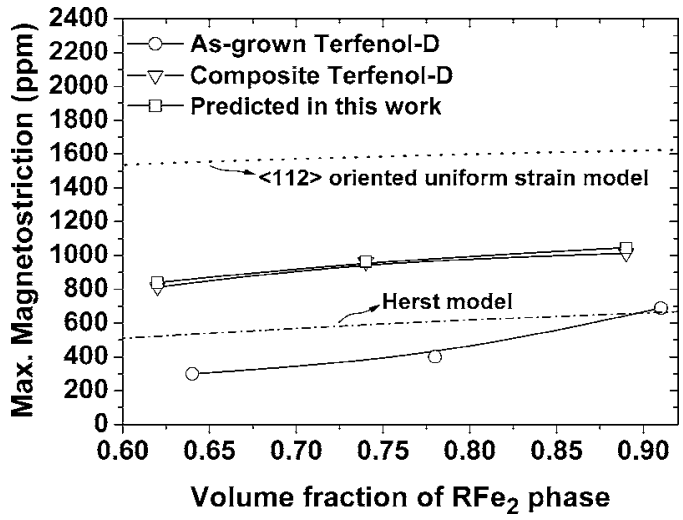

FIG. 3. Maximum magnetostriction and modeled results of the magnetostrictive strain calculated with the modified uniform strain condition considering the texture of the $R \mathrm{Fe}_{2}$ phase and change in the elastic modulus with texture. The dotted line was calculated under the assumption that the magnetostrictive phase was oriented along the $\langle 112\rangle$ direction. The dash-dotted line was calculated with the aid of the Herbst model (see Ref. 8), which assumes an elastically isotropic magnetostrictive sphere. The maximum magnetostriction was obtained at $80 \mathrm{kA} / \mathrm{m}$ and a preload of $8 \mathrm{MPa}$. direction, which has a saturation magnetostriction of $1640 \mathrm{ppm}$ (Ref. 20) and that the elastic modulus of the magnetostrictive phase is $30 \mathrm{GPa}$ (the mean value calculated from the wide range of stiffness values reported for Terfenol-D from 25 to $35 \mathrm{GPa}$ ). This uniform strain model indicates a major discrepancy compared to the experimental results for grain-aligned composite Terfenol-D. This suggests that the grain-aligned composite Terfenol-D produced using the crystal growth technique should consider the preferred direction of the magnetostrictive phase for grain-aligned composite Terfenol-D when determining the texture properties fabricated using zone melting method.

In this study, since our modified uniform strain model considered the change in the elastic modulus related to the crystal direction of the magnetostrictive phase, the predicted magnetostriction of the composite was in good agreement with the experimental results.

\section{CONCLUSION}

In conclusion, we propose a model for predicting the effective magnetostriction of grain-aligned composite Terfenol-D, utilizing a modified uniform strain model that considers the elastic modulus coupled in the preferred direction. The predicted magnetostriction of grain-aligned composite Terfenol-D showed good agreement with the experimental results. Since our methodology accounts for the preferred direction of the unidirectionally grown crystal and the elastic modulus change related to each crystal direction, it can be used to assess the influences of different contributions on the effective magnetostriction. Therefore, this model can provide criteria for choosing the best combination of constituent materials for high-frequency magnetostrictive transducers or sensors. Furthermore, we confirmed that the effective magnetostriction of grain-aligned composite Terfenol-D depends on the preferred direction of the $R \mathrm{Fe}_{2}$ phase.

${ }^{1}$ L. Sandlund, M. Fahlander, T. Cedell, A. E. Clark, J. B. Restorff, and M. Wun-Fogle, J. Appl. Phys. 75, 5656 (1994).

${ }^{2}$ Z. J. Guo, S. C. Busbridge, Z. D. Zhang, B. W. Wang, and A. R. Piercy, J. Magn. Magn. Mater. 239, 554 (2002).

${ }^{3}$ S. W. Or, N. Nersessian, G. P. McKnight, and G. P. Carman, J. Appl. Phys. 93, 8510 (2003).

${ }^{4}$ D. Brugel, M. R. J. Gibbs, and P. T. Squire, J. Appl. Phys. 75, 4249 (1988).

${ }^{5}$ L. Ruiz de Angulo, J. S. Abell, and I. R. Harris, J. Magn. Magn. Mater. 157, 508 (1996) 
${ }^{6}$ O. Y. Kwon, J. C. Kim, Y. D. Kwon, D. J. Yang, S. H. Lee, Z. H. Lee, and S. H. Hong, Appl. Phys. A: Mater. Sci. Process. 80, 1563 (2005).

${ }^{7}$ O. Y. Kwon, H. Y. Kim, S. I. Cha, and S. H. Hong, J. Appl. Phys. 97, 113904 (2005).

${ }^{8}$ J. F. Herbst, T. W. Capehart, and F. E. Pinkerton, Appl. Phys. Lett. 70, 3041 (1997).

${ }^{9}$ C. W. Nan, Appl. Phys. Lett. 72, 2897 (1998).

${ }^{10}$ Y. Chen, J. E. Snyder, C. R. Schwichtenberg, K. W. Dennis, and D. K. Falzgraf, Appl. Phys. Lett. 74, 1159 (1999).

${ }^{11}$ Z. J. Guo, S. C. Busbridge, A. R. Piercy, Z. D. Zhang, X. G. Zhao, and B. W. Wang, Appl. Phys. Lett. 78, 3490 (2001).

${ }^{12}$ H. J. Bunge, in Preferred Orientation in Deformed Metals and Rocks: An Introduction to Modern Texture Analysis, edited by H.-R. Wenk (Academic, London, 1985), pp. 73-108.

${ }^{13}$ J. F. Nye, Physical Properties of Crystals (J. W. Arrowsmith, London,
1985), p. 131.

${ }^{14}$ O. Y. Kwon, H. Y. Kim, S. I. Cha, and S. H. Hong, J. Appl. Phys. 97, 113905 (2005).

${ }^{15}$ B. Bhattacharya, B. R. Vidyashankar, S. Patsias, and G. R. Tomlinson, Proc. SPIE 204, 4073 (2000).

${ }^{16}$ C. W. Nan, Y. Huang, and G. J. Weng, J. Appl. Phys. 88, 339 (2000).

${ }^{17}$ J. D. Verhoeven, J. E. Ostenson, E. D. Gibson, and O. D. McMaster, J. Appl. Phys. 66, 772 (1989).

${ }^{18}$ W. Mei, T. Okane, and T. Umeda, J. Alloys Compd. 248, 132 (1997).

${ }^{19}$ J. P. Teter, M. Wun-Fogle, A. E. Clark, and K. Mahoney, J. Appl. Phys. 67, 5004 (1990).

${ }^{20}$ J. D. Verhoeven, E. D. Gibson, O. D. McMaster, and H. H. Baker, Metall. Trans. A 18A, 223 (1987).

${ }^{21}$ M. Zhang, X. Gao, S. Zhou, and Z. Shi, J. Alloys Compd. 385, 309 (2004). 